\title{
INFILTRAÇÃO DE ÁGUA EM SOLO, DETERMINADA POR SIMULADOR DE CHUVAS E PELO MÉTODO DOS ANÉIS ${ }^{1}$
}

\author{
Édio Luiz da Costa ${ }^{2}$, Antônio Marciano da Silva ${ }^{3}$, Alberto Colombo ${ }^{4}$ \& Agostinho R. de Abreu
}

\begin{abstract}
RESUMO
Devido à grande variabilidade que ocorre no processo de obtenção da capacidade de infiltração do solo, o método a ser adotado na sua obtenção deve ser o mais coerente possível com o sistema de irrigação a ser utilizado fornecendo, assim, dados confiáveis ao projeto. Avaliaram-se, neste trabalho, dois métodos de determinação da capacidade de infiltração, usando-se um infiltrômetro de anéis concêntricos e um de aspersão tipo simulador de chuvas, com o solo na capacidade de campo até a profundidade de $40 \mathrm{~cm}$, cujos resultados mostraram que a capacidade de infiltração determinada pelos anéis, que é o método mais usado, resulta em valores maiores que os determinados pelo simulador de chuvas.
\end{abstract}

Palavras-chave: simulador de chuvas, infiltração, velocidade de infiltração

\section{INFILTRATION CAPACITY DETERMINED BY RAINFALL SIMULATOR AND INFILTROMETER RINGS METHOD}

\begin{abstract}
Because of the high variability among the methods used to obtain soil infiltration capacity, the evaluation method to be used should be most consistent with the specific irrigation system, otherwise, the obtained information may not be reliable for designing. In this research, the "ring infiltrometer" and the "sprinkler infiltrometer" (rainfall simulator) methods were evaluated, for soil field-capacity moisture conditions. The results showed that ring infiltrometer method gives higher values for infiltration capacity than those obtained by the rainfall simulator.
\end{abstract}

Key words: rainfall simulator, infiltration, infiltration velocity

\section{INTRODUÇÃO}

Por envolver conhecimentos relativos ao solo, água, clima e planta, a irrigação é considerada uma tecnologia complexa. No dimensionamento de um projeto de irrigação é necessário que se tenha conhecimento sobre as características do solo, do clima e da planta. Das características do solo, a capacidade de infiltração é uma das principais, pois reflete a capacidade máxima que o solo tem em permitir a entrada de água no seu interior, sob determinadas condições, tornando-se num dos parâmetros mais importantes que afetam a irrigação. Segundo Scaloppi (1986) a infiltração do solo tem grande influência em todas as fases da

\footnotetext{
${ }^{1}$ Parte da dissertação do primeiro autor, apresentada à Universidade Federal de Lavras, MG - UFLA, para obtenção do título de Mestre em Engenharia Agrícola

${ }^{2}$ Pesquisador EPAMIG M.Sc. em Engenharia Agrícola. EPAMIG/CTNM, CP 12, CEP 39440 - 000, Janaúba, MG, Telefax: (038) 821 2160 E-mail: epamig@al.connect.com.br

${ }^{3}$ Prof. Titular Dr., Departamento de Engenharia da UFLA, CP 12, CEP 37200 - 000, Lavras, MG

${ }^{4}$ Prof. Assistente M.Sc. do Departamento de Engenharia da UFLA

${ }^{5}$ Prof. Adjunto Dr. do Departamento de Ciências Exatas da UFLA
} 
irrigação e, conseqüentemente, sobre o desempenho do sistema, sendo considerada, por Walker \& Skogerboe (1987) de difícil determinação, por causa de sua variabilidade espacial e temporal. São vários os fatores que afetam o processo de infiltração, dentre os quais se destacam: as condições da superfície do solo, o conteúdo inicial de água no perfil do solo, o tempo de infiltração, a existência de camadas menos permeáveis ao longo do perfil, a topografia do terreno e as propriedades físicas do solo (Bernardo, 1986; Daker, 1988; Hillel, 1970; Rezende \& Scaloppi, 1985). Segundo Moore \& Larson (1980) o encrostamento, ou selamento superficial, é um processo causado principalmente pelo impacto das gotas de chuva. A crosta superficial, embora sendo de pequena espessura, reduz significativamente a infiltração aumentando, conseqüentemente, o escoamento superficial. A avaliação da infiltração d'água no solo depende da escolha de um infiltrômetro adequado ao tipo de estudo que, por sua vez, irá depender do método de irrigação usado e da necessidade de serem obtidos dados condizentes com o sistema de irrigação escolhido. Os métodos mais usados para a determinação da infiltração, são: método de entrada e saída de água no sulco, anéis infiltrômetros e simuladores de chuva. $\mathrm{O}$ método dos sulcos de entrada e saída é de uso específico para o sistema de irrigação por sulcos. Segundo Vieira (1977) o infiltrômetro de anéis concêntricos é o mais usado, porém tem suas limitações, quando usado para fins de elaboração de projetos de irrigação por aspersão ou para trabalhos em que os dados gerados se dêem através de precipitações. Estudos revelam que os valores da capacidade de infiltração do solo, determinada pelos anéis infiltrômetros, são sempre maiores que os estabelecidos pelo simulador de chuvas. Os testes comparativos de infiltração com anéis concêntricos e o simulador de chuvas, realizados por (Horton,1940; Neal, citado por Baver, 1972; Sidiras \& Roth, 1987; Pruski et al., 1994) evidenciaram a diferença entre os dois métodos e concordaram que a causa deste fato era devido ao impacto direto das gotas com a superfície do solo, que ocorre no simulador e não ocorre nos anéis. Objetivou-se, neste trabalho, determinar as diferenças entre as velocidades de infiltração, usando-se um simulador de chuvas e um infiltrômetro de anéis concêntricos.

\section{MATERIAL E MÉTODOS}

O ensaio foi realizado na área de campo do Departamento de Engenharia da Universidade Federal de Lavras, Minas Gerais. O solo da área foi classificado como Latossolo Roxo distrófico (Lrd) (Andrade, 1979), horizonte A profundo, textura argilosa e relevo suave, com declividade de $10 \%$. O material originário é rocha eruptiva básica e este solo tem, como característica marcante, uma alta capacidade de infiltração, devido à sua estrutura , apesar de ser um solo com alto teor de argila (Rocha, 1982).

A determinação da infiltração foi feita com três repetições. Os teste foram realizados com o solo na umidade correspondente à capacidade de campo. $\mathrm{O}$ tamanho das parcelas usadas para os teste com o simulador de chuvas foram de $3 \times 1,2 \times 1 \mathrm{e} 1 \mathrm{x} 1 \mathrm{~m}$, devidamente limitadas por chapas de aço $\mathrm{n}^{-} 18$, de $25 \mathrm{~cm}$ de altura, dos quais $15 \mathrm{~cm}$ foram cravados no solo.

O simulador de chuvas usado foi desenvolvido por (Costa, 1996) e é semelhante a um sistema de irrigação linear móvel
(Figura 1) apresentando as seguintes características: intensidade de precipitação de $70 \mathrm{~mm} \mathrm{~h}^{-1}$, uniformidade de aplicação de água de $80 \%$, diâmetro médio de gotas incidentes sobre as parcelas de $0,84 \mathrm{~mm}$ e energia cinética de $66,43 \mathrm{~kJ} \mathrm{ha}^{-1} \mathrm{~mm}$, a uma pressão de serviço de $240 \mathrm{kPa}$, mantida constante com o uso de válvulas reguladoras de pressão. A alimentação de todo o conjunto foi feita por uma moto bomba que capta água de um reservatório de $5 \mathrm{~m}^{3}$, o qual era abastecido por outra moto bomba instalada próximo a uma represa; a água passava por um sistema de filtragem composto por um filtro de areia e outro de tela.

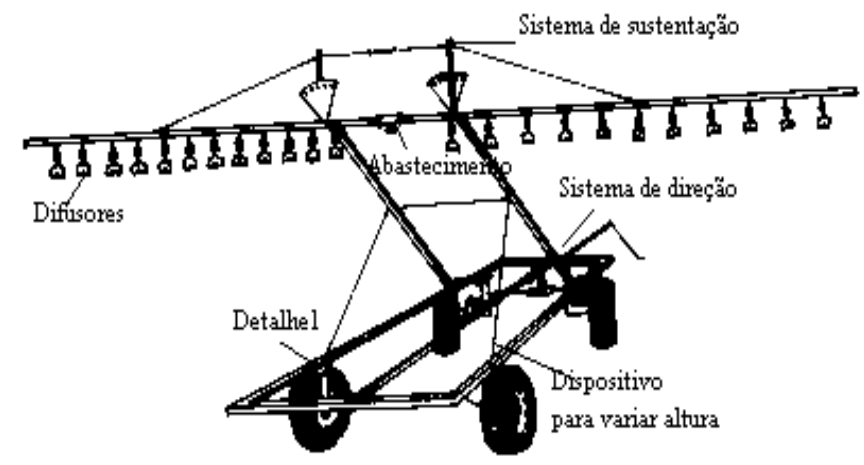

Figura 1. Visão geral do simulador de chuvas, componentes e acessórios

A determinação da infiltração foi feita pela diferença entre a lâmina de água precipitada sobre a parcela e a água escoada e coletada a jusante, numa calha instalada transversalmente e abaixo da superfície do terreno. Para captar a vazão efluente desta calha, uma mangueira "cristal", com comprimento de $4 \mathrm{~m}$ e $32 \mathrm{~mm}$ de diâmetro, foi conectada à sua saída permitindo, em cota mais baixa e fora do alcance da chuva simulada, o armazenamento da água do escoamento, em recipiente graduado de 20 L. Os testes de infiltração tiveram duração igual ou superior a $2 \mathrm{~h}$, sendo a coleta do escoamento superficial dividida em quatro intervalos de $5 \mathrm{~min}$, dois intervalos de $10 \mathrm{~min}$ e o restante em intervalos de $20 \mathrm{~min}$. As medidas do escoamento superficial foram feitas de forma direta, pela leitura de volume e tempo correspondente subtraindo-se, da chuva aplicada, o volume obtido no coletor, em determinado tempo, obtendo-se o volume infiltrado, o qual foi transformado em lâmina e dividido pela área da parcela.

Simultaneamente aos testes realizados com o simulador de chuvas, foram realizados testes com os anéis infiltrômetros, possibilitando a comparação entre os dados de infiltração obtidos por um método dinâmico e outro estático, simulador de chuvas e os anéis infiltrômetros, respectivamente. Para o teste com o infiltrômetro de anéis, foram usados dois anéis metálicos concêntricos, com diâmetro de 30 e $60 \mathrm{~cm}$, ambos com $30 \mathrm{~cm}$ de altura, cravados $15 \mathrm{~cm}$ no solo e nivelados de acordo com recomendações de Bernardo (1986). O sistema de medição da variação da altura d'água consistia de uma régua graduada em centímetros colocada no anel interno. Em todos os testes e em ambos os cilindros que compõem o infiltrômetro circular, o nível de água foi mantido o mesmo e igual a $8 \mathrm{~cm}$. A infiltração foi medida pela determinação da variação de leitura obtida na régua instalada no anel menor e o tempo necessário para que ocorresse tal variação, a qual, quando permitida, era no máximo igual a $2 \mathrm{~cm}$. Os ensaios com os anéis foram feitos nas mesmas 
condições de umidade e repetição dos testes realizados com o simulador de chuvas.

A comparação entre os métodos foi promovida pela análise de variância dos parâmetros n e $\mathrm{f}_{1}$ da equação de Kostiakov (Eq. 1) usada para estimar a capacidade de infiltração do solo (f).

$$
\mathrm{f}=\mathrm{f}_{1} \cdot \mathrm{t}^{\mathrm{n}}
$$

em que:

f - capacidade de infiltração;

$\mathrm{f}_{1} \quad$ - capacidade de infiltração no tempo $\mathrm{t}=1$;

n - expoente da equação e

t $\quad$ - tempo de infiltração considerado.

A Eq. 1 foi obtida por derivação da equação de infiltração acumulada (Eq. 2) que foi ajustada a partir dos dados obtidos.

em que:

$$
\mathrm{F}=\mathrm{F}_{1} \cdot \mathrm{t}^{\mathrm{m}}
$$

F - infiltração acumulada;

$\mathrm{F}_{1}$ - infiltração acumulada no tempo $\mathrm{t}=1$ e $\mathrm{m}=$ expoente da equação.

Determinou-se também, a capacidade de infiltração básica $(f \infty)$, pela Eq. 3 .

$$
f_{\infty}=f_{1} \cdot\left(\frac{\tan \alpha}{-n \cdot f_{1}}\right)^{\frac{-n}{-n-1}}
$$

em que:

$f \infty$ - capacidade de infiltração básica

$\tan \alpha--0,01$, que corresponde a $\alpha=179$ e $\alpha=$ ângulo entre a curva e o eixo dos tempos.

\section{RESULTADOS E DISCUSSÃO}

O LRd é um solo extremamente argiloso (cerca de $60 \%$ de argila ao longo do perfil) o que seria fator limitante à capacidade de infiltração; isto, no entanto, não aconteceu devido à estrutura granular apresentada por este tipo de solo, que favorece o processo de infiltração. A elevada porosidade apresentada pelo solo, acima de $60 \%$, com adequada proporção de macroporos, cerca de $1 / 3$, confere-lhe elevado valor de permeabilidade e condutividade hidráulica (Tabela 1).

Pelo fato do método do simulador de chuvas ter sido utilizado para diferentes comprimentos das parcelas e o dos anéis não permitir o mesmo, os dados foram reunidos e deles se extraiu a média. Os resultados estão mostrados na Tabela 2.

\begin{tabular}{|c|c|c|c|c|c|c|c|c|}
\hline \multirow[t]{2}{*}{ Repetição } & \multicolumn{4}{|c|}{ Simulador de chuvas } & \multicolumn{4}{|c|}{ Anéis concêntricos } \\
\hline & $f_{1}$ & $\mathrm{n}$ & $\mathrm{f}_{\infty} \mathrm{mm} \mathrm{min}^{-1}$ & $\mathrm{R}^{2}$ & $\mathrm{f}_{1}$ & $\mathrm{n}$ & $\mathrm{f}_{\infty} \mathrm{mm} \mathrm{min}^{-1}$ & $\mathrm{R}^{2}$ \\
\hline $\mathrm{R} 1$ & 1,037 & $-0,29$ & 0,355 & 0,95 & 1,288 & $-0,48$ & 0,187 & 0,98 \\
\hline $\mathrm{R} 2$ & 1,123 & $-0,26$ & 0,276 & 0,99 & 2,335 & $-0,19$ & 0,958 & 0,99 \\
\hline R3 & 1,672 & $-0,20$ & 0,464 & 0,99 & 3,323 & $-0,20$ & 1,215 & 0,99 \\
\hline Média & 1,277 & $-0,25$ & 0,365 & ----- & 2,315 & $-0,29$ & 0,787 & ----- \\
\hline $\mathrm{T}_{\mathrm{f} \infty}(\min )$ & & & 97,6 & & & & 155,4 & \\
\hline
\end{tabular}

Tabela 2. Valores dos parâmetros da equação de Kostiakov para o simulador de chuvas e os anéis

A análise de variância para os parâmetros $\left(f_{1} e n\right)$ das equações geradas com os dados obtidos com o simulador e com os anéis concêntricos, não mostrou efeito significativo do método para o expoente $n$; no entanto, o coeficiente $\mathrm{f}_{1}$ mostrou diferença significativa a nível de $17 \%$, valor este aceitável devido à grande variabilidade que existe nos solos para a capacidade de infiltração. O resultado da análise estatística veio confirmar que $\mathrm{n}$ não é afetado pelos métodos, pois é um parâmetro que depende das características do solo e já $\mathrm{f}_{1}$ é afetado, pois este representa a infiltração do solo, que se dá de forma diferenciada, devido às características de aplicação de cada infiltrômetro. Comparando-se os valores médios do coeficiente fo obtidos para os dois métodos, encontra-se uma proporção de 1:2,15 vezes, respectivamente, entre o simulador e os anéis. Este resultado é semelhante aos encontrados por Neal, citado por Baver (1972) que foi de duas a três vezes maior, concordando também com os trabalhos de Horton (1940) e Pruski et al. (1994). A capacidade de infiltração básica não se manteve constante em todos os ensaios, tanto para o simulador quanto para os cilindros, em razão, certamente, da variabilidade espacial entre as áreas amostradas. As diferenças encontradas nos resultados mostram que os anéis superestimam os valores de infiltração no caso da aspersão. As curvas de capacidade de infiltração determinadas pelos dois métodos estão representadas na Figura 2.

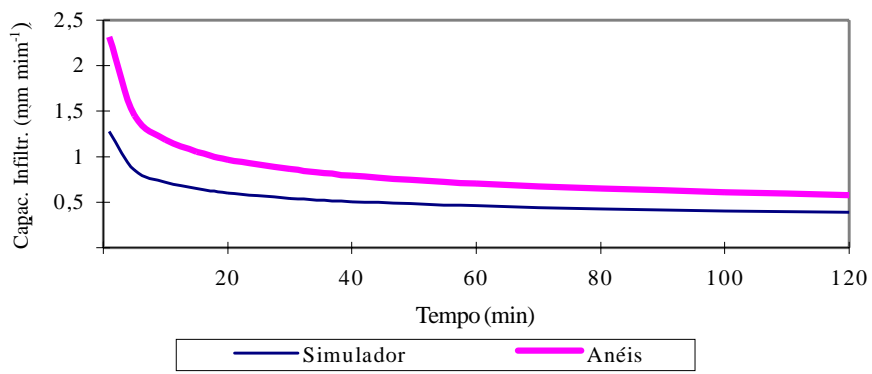

Figura 2. Curvas de capacidade de infiltração determinadas pelo simulador de chuvas e pelos anéis concêntricos para o

\begin{tabular}{|c|c|c|c|c|c|c|c|c|}
\hline \multirow{2}{*}{$\begin{array}{l}\text { Repetição } \\
\text { Prof. - cm }\end{array}$} & \multicolumn{3}{|c|}{ Granulometria } & \multicolumn{2}{|c|}{ Densidade } & \multicolumn{3}{|c|}{ Porosidade } \\
\hline & Argila \% & Areia $\%$ & Silte $\%$ & $\mathrm{dp}\left(\mathrm{g} \mathrm{cm}^{-3}\right)$ & $\mathrm{dg}\left(\mathrm{g} \mathrm{cm}^{-3}\right)$ & VTP \% & Micro $\%$ & Macro \% \\
\hline R1 $0-20$ & 64,00 & 12,67 & 23,33 & 2,86 & 0,97 & 66,08 & 39,80 & 26,28 \\
\hline R2 $0-20$ & 65,00 & 12,00 & 22,67 & 2,86 & 1,09 & 61,85 & 39,75 & 22,10 \\
\hline $20-40$ & 67,00 & 12,00 & 20,67 & 2,86 & 0,86 & 70,00 & 39,83 & 30,17 \\
\hline $20-40$ & 64,00 & 12,67 & 23,00 & 2,86 & 1,11 & 61,31 & 40,82 & 20,49 \\
\hline
\end{tabular}
LRd - Lavras, 1996

Tabela 1. Características físicas do Latossolo Roxo distrófico da área em estudo

${ }^{1}$ Análises realizadas no Laboratório de Solos do Departamento de Ciências do Solo/UFLA-Lavras, MG 
Verifica-se que, para os mesmos valores de tempo, a capacidade de infiltração determinada pelos anéis é superior àquela indicada pelo simulador, cuja explicação deve à forma de aplicação da água sobre a superfície. Nos anéis concêntricos, por manter uma carga hidráulica sobre a superfície, a infiltração ocorre na taxa máxima que o solo permite, sendo restringida apenas pelas caraterísticas do próprio solo, que não sofre nenhuma modificação em sua superfície, favorecendo altas infiltrações; já no simulador de chuvas não existe uma lâmina de água sobre a superfície do solo, ficando o mesmo sempre exposto à ação direta do impacto das gotas da chuva e do efeito do escoamento superficial. Esses fatores levam à desagregação das partículas do solo, que podem ser carreadas pela enxurrada, arremessadas pelo salpico ou, ainda, deslocadas para o interior do solo, formando uma crosta superficial, com maior densidade, fazendo diminuir a intensidade de infiltração da água no solo.

$\mathrm{Na}$ Tabela 1 tem-se os tempos estimados para que a capacidade de infiltração básica seja alcançada. Nota-se que, usando-se anéis concêntricos, é necessário um tempo muito longo, em média 155,4 min, devido ao método utilizado e à alta capacidade de infiltração dos Latossolos Roxos e, em particular, do LRd estudado; já para o método do simulador de chuvas verifica-se que o tempo é bem menor que o encontrado pelos anéis, em média 97,6 min, cuja diferença é também explicada pelos motivos já apresentados.

\section{CONCLUSÕES}

A partir dos dados encontrados, das referidas análises e para as condições em que o trabalho foi realizado, chegou-se às seguintes conclusões:

1. Os métodos fornecem diferentes valores para os parâmetros da equação de Kostiakov, sendo significativa a influência sobre o coeficiente $\mathrm{f}_{1}$, a nível de $17 \%$, e não significativa sobre o expoente $\mathrm{n}$.

2. O valor da capacidade de infiltração determinada pelo simulador de chuvas é menor que o dos anéis infiltrômetros, devido à ação combinada da chuva e do escoamento superficial, indicando um cuidado a ser observado, quando da escolha do tipo de infiltrômetro a ser usado, na obtenção de dados para elaboração de projetos de irrigação; com isto, recomenda-se, para projetos de irrigação por aspersão, utilizar o simulador de chuvas.

\section{REFERÊNCIAS BIBLIOGRÁFICAS}

ANDRADE, H. Caracterização genética e morfológica e classificação de dois solos do município de Lavras, MG, em correlação com a geomorfologia da área. Lavras: ESAL, 1979. 84p. Dissertação Mestrado

BAVER, L.B. Soil physics. 4 ed. New York: J. Willey, 1972. 498p.

BERNARDO, S. Manual de irrigação. Viçosa: UFV, 1986. 596p.

COSTA, E.L. da. Desenvolvimento e avaliação de um simulador de chuvas para estudos de infiltração de água no solo. Lavras: UFLA, 1996. 78p. Dissertação Mestrado

DAKER, A. Irrigação e Drenagem; a água na agricultura. 7 ed. Rio de Janeiro: Freitas Bastos, 1988. v.3.543p.

HILLEL, D. Solo e Água; fenômeno e princípios físicos. Porto Alegre: UFRGS, 1970. 231p.

HORTON, R.E. An approach toward a physical interpretation capacity. Soil Science Society of America Proceeding, Madison, v.5, p.399-417, 1940.

MOORE, I.D.; LARSON, C.L. An infiltration - runoff model for cultivated of soils. Transactions of the ASAE, St. Joseph. v.23, n.6, p. 1460-1467, 1980.

PRUSKI, F.F.; VENDRADE, V.; OLIVEIRA, E.F. de; BALBINO, L.C.; FERREIRA, P.A.; WERLANG, L.; CARVALHO, L.T. de. Velocidades de infiltração da água em um Latossolo Roxo, determinadas com o simulador de chuvas e o infiltrômetro de anel, em ensaios sucessivos. In: CONGRESSO BRASILEIRO DE ENGENHARIA AGRÍCOLA, 23., 1994, Campinas. Resumos... Campinas: UNICAMP, 1994. p.189.

REZENDE, F.C.; SCALLOPI, J.E. Avaliação de métodos para a determinação das características de infiltração por sulcos. Pesquisa Agropecuária Brasileira, Brasília, v.20, n.2, p.227-233, 1985.

ROCHA, G.C. Geologia, geomorfologia e pedologia de uma catena de solos, situada no Campus da Escola Superior de Lavras, MG. Lavras: ESALQ, 1982. 109p. Dissertação Mestrado

SCALOPPI, E.J. Sistemas de irrigação por Superfície. Informe Agropecuário, Belo Horizonte, v.139, n.12, p.12-26, 1986.

SIDIRAS, N.; ROTH, C.H. Infiltration measurements with double-ring infiltrometers and a simulator under different surface conditions on an Oxisol. Soil and Tillage Research, Amsterdam, v. 9, p.161-168, 1987.

VIEIRA, H.A. Comportamento de quatro infiltrômetros na determinação da velocidade de infiltração básica d'água no solo. Viçosa: UFV, 1977. 52p. Dissertação Mestrado

WALKER, W.R.; SKOGERBOE, G.V. Teoria y practica del riego por superfície. Logan: International Irrigation Center, 1987. 459p. 